\title{
Correction to: MicroSCOPE: Enabling Access Control in Searchable Encryption with the Use of Attribute-Based Encryption and SGX
}

\author{
Antonis Michalas, Alexandros Bakas, Hai-Van Dang, \\ and Alexandr Zalitko
}

\author{
Correction to: \\ Chapter "MicroSCOPE: Enabling Access Control \\ in Searchable Encryption with the Use of Attribute-Based \\ Encryption and SGX" in: A. Askarov et al. (Eds.): \\ Secure IT Systems, LNCS 11875, \\ https://doi.org/10.1007/978-3-030-35055-0_16
}

The original version of this chapter contained an error in the fourth author's name. The spelling of Alexandr Zalitko's name was incorrect in the header of the paper. The author name was corrected. 